\title{
Downlink Frequency Reuse and Hopping for OFDMA Femtocells
}

\begin{abstract}
To prevent the Femto-to-Macro and Femto-to-Femto inter-cell interference, it is essential for the Femto Base Stations to perform interference management in its downlink. This paper exploits the distinct characteristics of OFDMA Femtocells and reveals the simple but effective mechanics for Femto BSs to mitigate the inter-cell interference when Macro BSs are performing fractional frequency reuse. The idea consists of frequency pattern counter-reusing and band-wide frequency hopping. Base on this idea, we then proposed a heuristic Frequency Reuse and Hopping scheme and the later simulation results proved that it can satisfactorily improve the system performance. By following the routines of low-cost low-complexity in design, the proposed scheme is grounded on realistic assumptions and easy to implement.
\end{abstract}

Index Terms - OFDMA, Femtocell downlink, Frequency Reuse, Frequency Hopping

\section{Introduction}

The future cellular networks witness an inevitable trend towards versatility and heterogeneity. To strengthen the cellular service in residential or small office/home office (SOHO) use, a smaller in size and more flexible in deployment, namely "Femto Cellular Network" (Femtocell) or "Femto/Home Base Station" (Femto BS/HBS) has been recently proposed [1]. With such a low-power base station (BS) installed in house, customer can enjoy enhanced signal coverage and improved Quality of Service (QoS). Many providers are of confidence that Femtocells or similar products will play a significant role in improving the business cases and realizing the full potentials of long term evolution (LTE) [2].

While facilitating the mobile-nomadic convergence at home, embedding Femtocells into conventional cellular architecture (Macrocell) also brings challenges to research. One of the most notable technical challenges is how to manage the interference. Femtocells and Macrocells most likely share the same licensed spectrum of their providers for economic reasons. Such system is named by research as twotier cellular network [4]. As an underlying secondary user, Femtocells, although mostly deployed indoors, can sometimes cause serious inter-cell interference due to no prior network planning. As shown by the interference study report from Femto Forum, downlink Femto-to-Macro (cross-tier) interference can have a high influence level and sometimes dramatically deteriorate the system performance [3]. Additionally, when the number of Femtocells grows high, the Femto-to-Femto (2nd-tier) interference also becomes significant that degrades the system capacity.

Following the above introduction, this paper focuses on the design of Femtocell-dedicated OFDMA downlink (DL) transmission schemes. To be more specific, we will consider a Macrocell system adopting Soft (SFR) or Fractional Frequency Reuse (FFR) in its DL. In such environment, we exploit the key characteristics of Femtocells and generate heuristic schemes to improve the performance. Hereby simplified schemes are favored due to the strict restrictions in costs and hardware for Femtocell implementation. It is not yet possible for the current Femtocells to realize extensive synchronization and coordination among each other (e.g. X2 interface in LTE) [5].

This paper is organized as follows. In Section 1.1, we will briefly introduce some related works. In Section 2, we will describe the system models and abstract the key characteristics of Femtocells. In Section 3, we will deliver the proposed scheme of design. In Section 4, we will introduce the methodologies of simulation and illustrate the simulation results. In Section 5, we give concluding remarks and discuss the future works.

\subsection{Related works}

For LTE and other OFDMA based mobile communication systems, where intra-cell interference is often benign, numerous efforts have been devoted to combating inter-cell interference. The classic approaches are comprised of intercell interference coordination (ICIC) schemes [11] [12] such as dynamic Soft Frequency Reuse, co-scheduling, or beamforming, which can be realized and substantially improve the system area spectrum efficiency (ASE). Moreover, many uncoordinated schemes are also proposed, such as [13] [14] applying to standalone situations where the peer network elements are difficult to coordinate. When tangling the interference problem in two-tier networks, some works such as [6-10] have been published in recently years. In [6], the authors gave basic guidelines of co-channel and orthogonal assignments for spectrum allocation in Femtocell application. In [9], the authors gave strong analytical proofs and proposed a decentralized spectrum allocation scheme depending on the per-tier throughputs and loading of users. It assumes a two-tier orthogonal access of spectrum and central hexagonal topology of Macrocells. In this paper, we follow [6]'s co-channel assignment and [9]'s method of modeling Femtocells as indoor hotspots. Innovatively, we consider a 3-sector Macrosite model and the specific case that Macrocells are adopting FFR or SFR schemes in their DL. The heuristics scheme we propose, although very simple, can successfully adapt itself to the Femtocell's characteristics and greatly improve the performance.

\section{System Models}

\subsection{Femtocell Descriptions}

As shown in Fig. 1, unlike the concept of Relay (inband backhaul), the Femtocells in this paper are assumed to backboned to Internet via xDSL or other connections. We assume the Femtocells to inherit the same air interface of the existed Macrocell, thus to be compatible with most of the user equipments (UE) in current market. As mentioned in Section 1, we also assume that Femtocells are quasi-standalone. Frequent signaling exchange between cells is not achievable, but they do can obtain some mid or long-term information by inquiry on Internet. Moreover, due to the cost limit, we assume no extra receiver set is available at Femto BS, so it is 


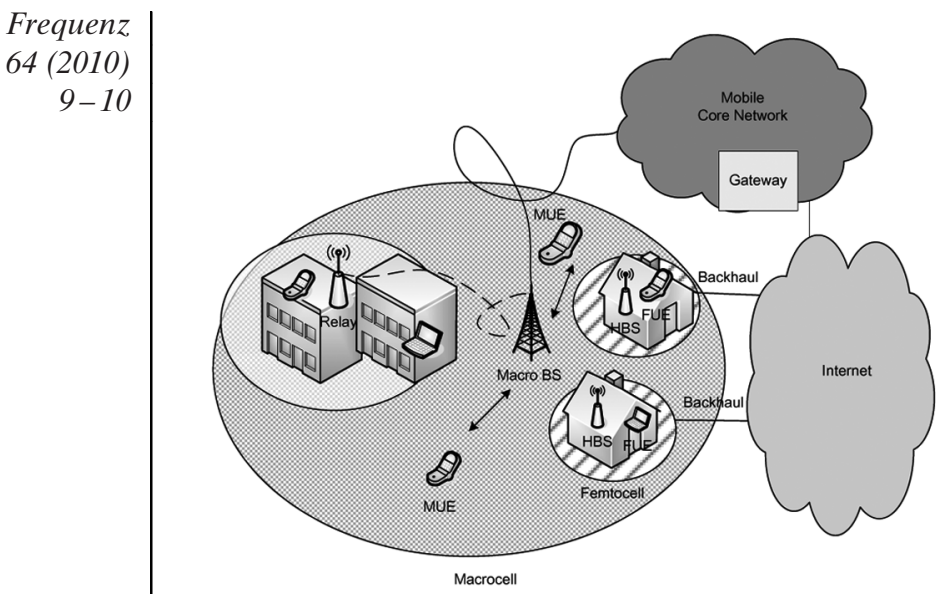

Fig. 1: The Concept of Femtocell

unable to listen on the DL control channel of neighboring cells to perform smart opportunistic scheduling. Nevertheless, by the feedback of channel quality information (CQI) from UE or sniffing on the random access or preamble signal channel in neighboring cell uplink (UL), where asynchronous receiving can be possible, Femtocells may have the opportunities to detect the approximate existence of victim BSs or UEs. Additionally, we make following assumptions to simplify the analysis:

AS1: FBS and FUE are always located indoors, distributed independently to the location of MBS and MUE.

We can notice that, compared to conventional Macrocells, Femtocells we modeled in this paper possess the following distinctions:

1. other than one-to-many communication, Femtocell DL is more likely to be one-to-few or even one-to-one,

2. the traffic demand from one Femtocell DL is much lighter than that of a Macro one,

3. due to small in size, the users in Femtocells are more symmetrically distributed

4. being deployed indoors, the interference leaked to the neighboring Macrocell or Femtocell takes wall and floor penetration.

\subsection{Basic System Assumptions}

As shown in Fig. 2, we assume a typical 3-sector layout for the Macrocell, with a total number of $M$ cells (i.e. $M / 3$ sites) in the system. We denote $m$ as the index of each Marcocell so $m=1, \ldots, M$. We further assume a total number of $N$ Femtocells (denoted as $n=1, \ldots, N$ ) are deployed in the system. As a scheme of universal frequency reuse, all the Macrocells and Femtocells are sharing the same spectrum. We assume that all UEs are one-to-one either connected to a Macrocell or a Femtocell. We denote them as $\mathrm{MUE}_{\mathrm{m}, \mathrm{i}}$ and $\mathrm{FUE}_{\mathrm{n}, \mathrm{i}}$ respectively, where $m$ and $n$ are the index of cell that UE is connected to and $i$ is the index of $\mathrm{UE}$ within the corresponding cell.

Furthermore, we assume a typical OFDMA system with total bandwidth of $20 \mathrm{MHz}$ in DL. The frequency band is divided into the amount of 100 subchannels (PRB), with each PRB contains 12 OFDM subcarriers. The system adopts typical OFDMA multi-access schemes to assign orthogonal resources in PRB wise to different users, and time is slotted.

By this definition, we assume that the communication link is only corrupted by thermal noise and the inter-cell interference. Hence we derive the receiving signal to noise and interference ratio (SINR) on subcarrier $j$ with:

$\operatorname{SINR}_{j}=\frac{P}{P_{\text {noise }}+\sum_{m, n} \bar{I}}$

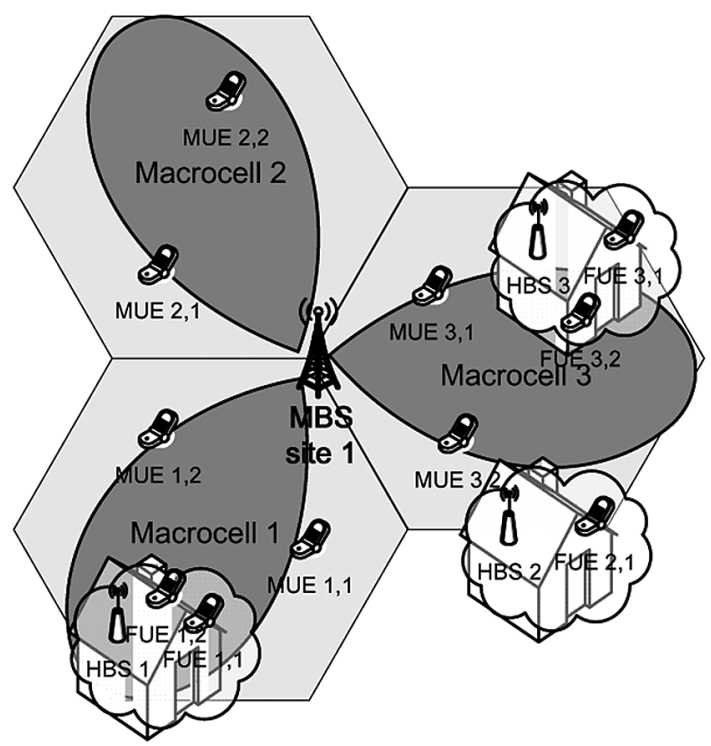

Fig. 2: Cell Layout with Macrocell and Femtocell

where $P$ and $P_{\text {noise }}$ is the power of receiving signal and noise. The averaged inter-cell channel interference $\bar{I}$ can appear when,

1. two (quasi)-synchronous Femto or Macro BSs are sending signals in the same subcarrier, or

2. two asynchronous Femto or Macro BSs with timing offset $\Delta t$ are sending signals in the adjacent subcarrier.

To be more specific, if we denote $T_{c p}$ as the duration of cyclic prefix (CP) and $T_{\text {OFDM }}$ as the duration of OFDM symbol, the interference suffered by the victim of $l$ subcarriers in distance can be calculated as, [15]

$$
\begin{aligned}
& I(\Delta t, l)= \\
& \left\{\begin{array}{cc}
\delta(l) & 0<\Delta \mathrm{t}<T_{c p} \\
\left|\frac{\sin \left(\pi l\left(T_{c p}-\Delta t\right) / T_{\text {OFDM }}\right)}{\pi l}\right|^{2} & T_{c p}<\Delta \mathrm{t}<T_{\text {OFDM }}+T_{c p}
\end{array}\right.
\end{aligned}
$$

\section{Heuristic Scheme: Frequency Reuse and Hopping}

\subsection{Frequency Reuse}

For many of the current and future OFDMA systems, it is very common to adopt FFR or SFR schemes in DL to better improve the system capacity. In FFR, the whole spectrum band $F$ is divided into three fractional sub-bands, with each sub-band $F_{i}, i=1,2,3$ contains $\left|F_{i}\right|$ subchannels respectively ${ }^{1}$. Simply we have $|F|=\left|F_{1}\right|+\left|F_{2}\right|+\left|F_{3}\right|$ and MUEs are categorized into two subsets, namely cell-center $C_{m}$ or cell-edge $E_{m}$ subsets, by distance or path loss estimation. Neighboring Macrocells reuse one of the sub-bands with factor of three to accommodate the cell-edge MUEs $\left\{M U E_{m, i} \mid M U E_{m, i} \in E_{m}\right\}$, which are more vulnerable to the other-cell interference. In the mean time, the cell-center MUEs are more robust to other-cell interference and can be allocated with channels of the whole band by reuse factor of one [16]. Therefore, the FFR edge pattern of each Macrocell can be depicted as in the left part of Fig. 3.

$\overline{1} F=F_{1} \cup F_{2} \cup F_{3}$, and we denote $\|$ as the number of subchannels of the band 


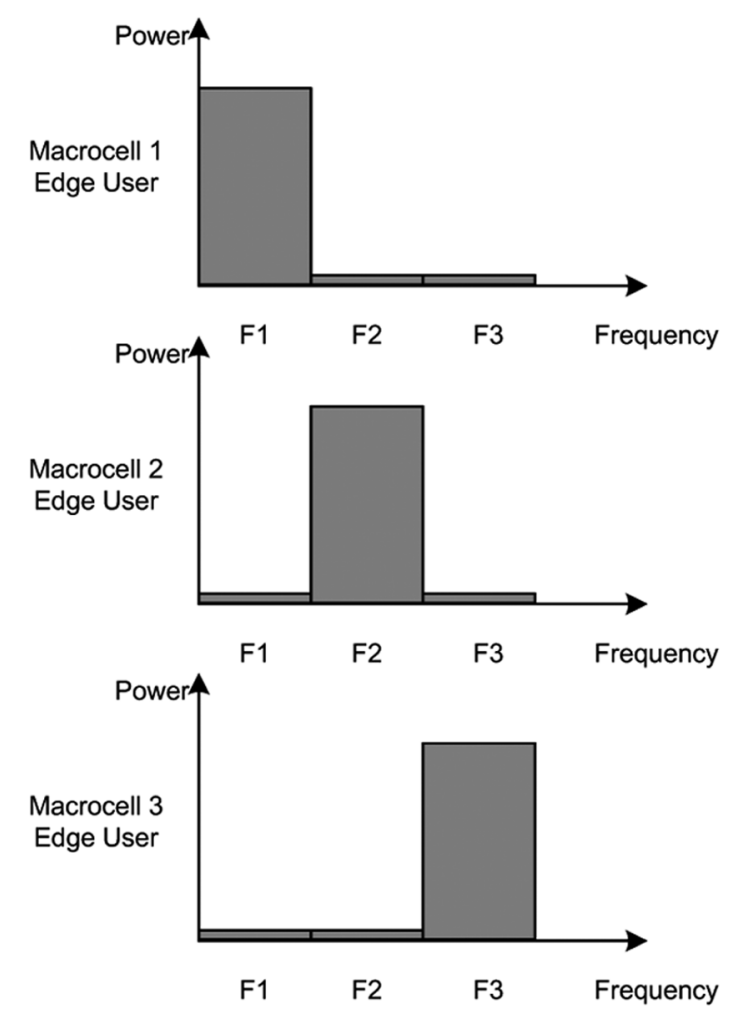

Fig.3: FFR in Macrocell and proposed Femtocell FFR

AS2: Each FBS $n$ is assumed to have the basic knowledge of its environment, knowing which is nearest Macrocell it is neighboring to (we denote it as $m_{n}$ ), cell-center or edge region it is belonging to, and what is the current FFR pattern for this Macrocell.

In such context, we give a proposition that it is always suboptimal for $n$ to reuse the frequency band that is the complement of the edge pattern of $m_{n}$. For instance, when $F_{1}$ is the edge pattern of $m_{n}$, the sub-optimal Frequency Reuse strategy for $n$ is $F \backslash F_{1}$, namely $F_{2} \cup F_{3}$. We name this complement as pattern counter-reusing.

The proposition is supported by the following two reasons:

Firstly, it is observed that Femtocells shall reuse the frequency of cell-edge MUEs as less as possible to avoid causing the co-channel interference to the Macrocell links, because this is the most malicious cross-tier interference since cell-edge MUEs are mostly vulnerable in link condition. On the other hand, the cell-center MUEs are robust to the interference from Femtocell since they are in good signal coverage from nearby MBS. Therefore, regardless cell-center or edge region the FBS is located, it is always "safe" to counter-reuse the cell-edge sub-band.

Secondly, compared to the macrocell, Femtocell user are more symmetrically distributed which means a minimal power control in Femtocell DL can be well adapted. In the mean time, due to the indoor-to-outdoor penetration, Femtocells' low adapted transmit power can hardly leak to the neighboring Femtocell. This is also supported by the study report of Femto Forum. [3]

This proposition makes it reasonable for the Femtocells to just adopt a simple counter-reusing policy when it has the knowledge of local Macrocell's pattern, regardless Macro cell center or edge region it is located. The heuristic counterreusing pattern policy is depicted in the right part of Fig. 3.

As an extension of FFR, SFR gives more degrees of freedom to the scheduler by only deploying a power mask for different neighboring cells. The counter-reusing pattern policy can therefore also be extended to facilitate the SFR schemes. The only difference to FFR is that, the sub-optimal strategy
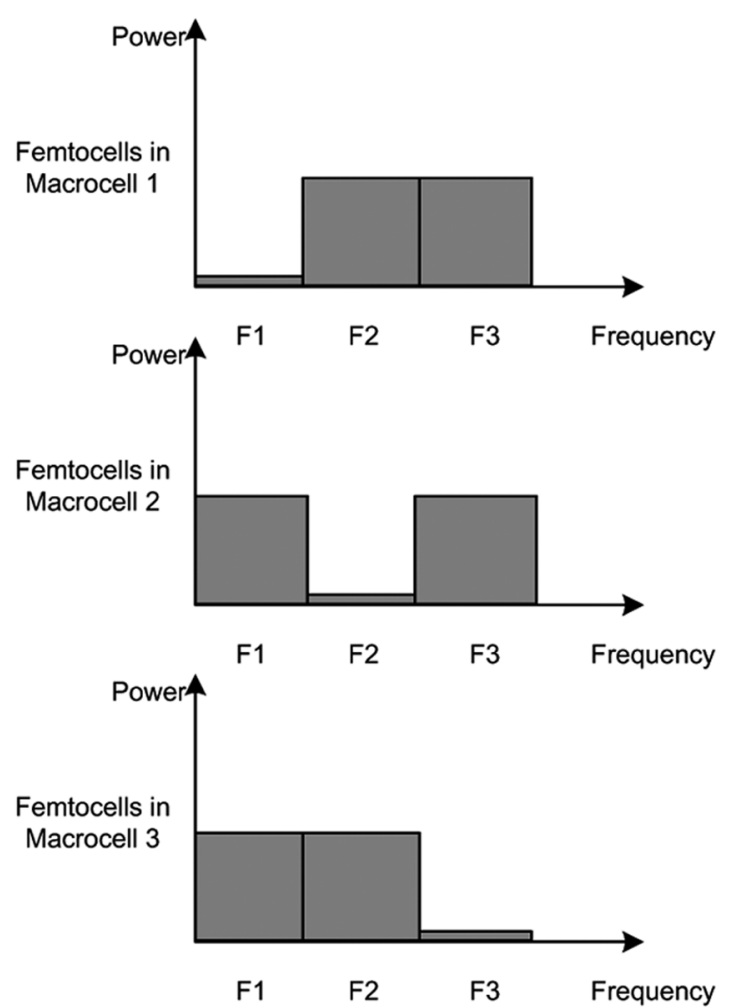

for Femtocell $n$ is the complement of the Macrocell's current cell-edge power mask instead of the specific sub-band $F_{i}$, which is depicted in Fig. 4.

\subsection{Frequency Hopping}

Downlink Frequency Hopping (FH) is not commonly adopted by LTE or others, mainly due to the fact that traffic load in $\mathrm{DL}$ is often crowded for such a one-to-many communication scenario. FH cannot benefit much in interference mitigation if the BSs are sending signal on all of its subchannels for all the time. On the contrary, near-far effect is very evident in cellular UL case. For such case, since normally only a small portion of frequency band demand is occupied by a single UE, there leaves a very wide range of spectrum diversity to exploit. For this reason, $\mathrm{FH}$ is frequently used in conventional mobile UL and effective in reducing the adverse influence of co-channel interference [16].

As described in Section 2.1, other than one-to-many, Femtocell DL is more similar to a one-to-one or one-to-few scenario with usually only a small portion of frequency demand. Therefore, the mechanics of FH in UL is also very beneficial to the case of Femto DL. Especially when Femtocells are pervasively deployed in great number, where Femto-to-Femto DL per-tier interference is becoming serious and limiting the system capacity. Only by the hopping schemes and randomizing the interference pattern, the mid-term experienced interference can be averaged to a lower level. By the laws of large number, FH in Femtocell DL thus significantly reduce the system outage rate. Therefore, we make the following assumption on the FBS FH functionality.

AS3: While Macrocell is not performing any FH in DL, each $F B S$ has the functionality to perform $F H$ in its $D L$ within the allocated sub-band and across them if allocated with several sub-bands. 


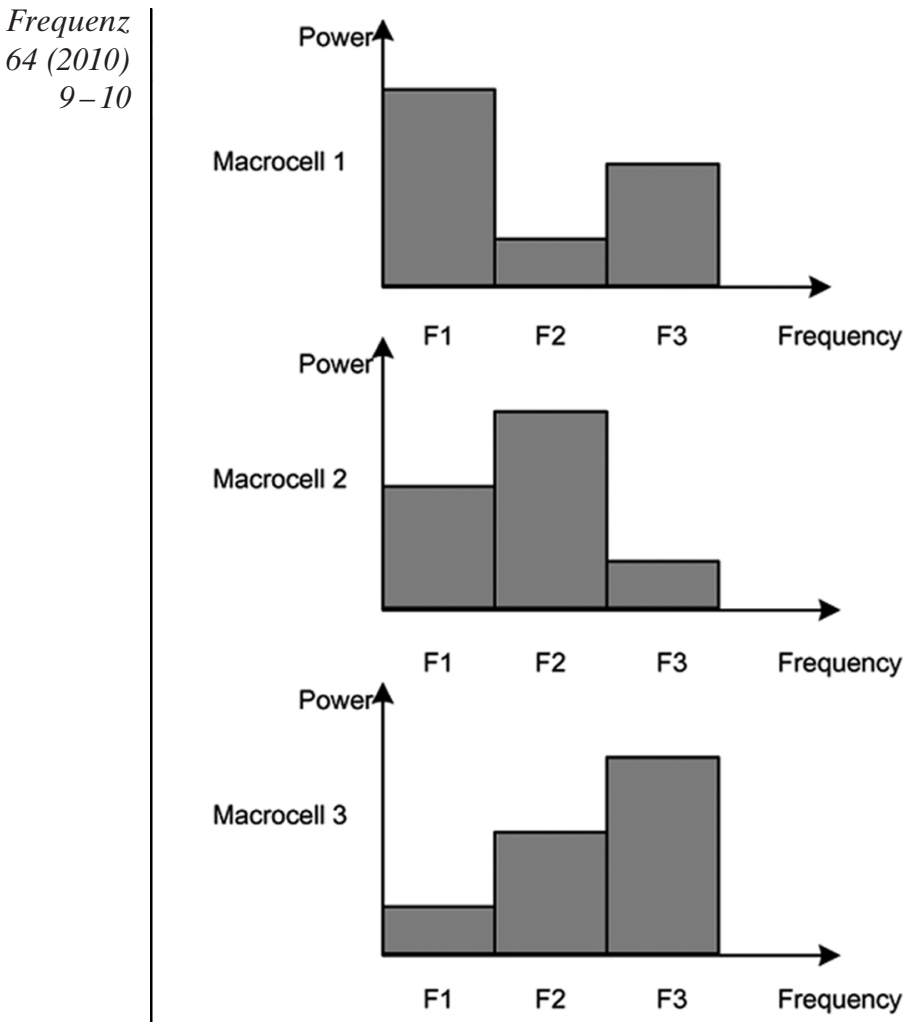

Fig.4: SFR power mask in Macrocell and proposed Femtocell SFR

Let the amount of demanded subchannels for Femtocell $n$ be $\left|f_{n}\right|$, which is a portion of $\rho_{n}=\frac{\left|f_{n}\right|}{\left|F_{1}\right|}$ to the width of sub-band ${ }^{2}$. Suppose neighboring Macrocell of Femtocell nis adopting FFR and the current cell edge pattern is $F_{3}$. According to the counter-reusing policy, $n$ is able to access $F_{1} \cup F_{2}$ with equal access probability. This applies also to the other Femtocells that share the same neighboring Macrocell as $n$. Apparently the best scheme for each of the Femtocell is to perform Frequency Hopping in the widest band possible, namely $F_{1} \cup F_{2}$. When $\rho_{n} \ll 1$, it dramatically cut the outage rate of two neighboring FBSs collide in two consecutive slots. And the $\mathrm{FH}$ can perform at even higher frequency if a high density of peer Femtocells are present in vicinity. Moreover, this FH scheme can also be extended to the SFR case, where the only difference is that, each Femtocell is accessing the sub-bands with unequal probability.

\subsection{Proposed Scheme}

By the analysis in the above sub-sections, we can conclude that FR counter-reusing is induced to mainly protect against the cross-tier interference and $\mathrm{FH}$ is performed to mitigate the Femto-to-Femto (2nd-tier) interference. Combining the above two ideas, we heuristically derive a joint Frequency Reuse and Hopping scheme for Femto DL communication. The proposed scheme can be described as follows:

Step 1: Periodically update neighboring Macrocell Frequency Reuse pattern by Internet inquiry

Step 2: if Macrocell FR pattern information unavailable,

2.1) define average transmit power by link feedback

2.2) select random hopping pattern

2.3) perform whole spectrum frequency hopping Step 3: if Macrocell FR pattern information available

3.1) define counter-reusing pattern and power mask

${ }^{2}$ For simplicity, we assume equal width for each sub-band.

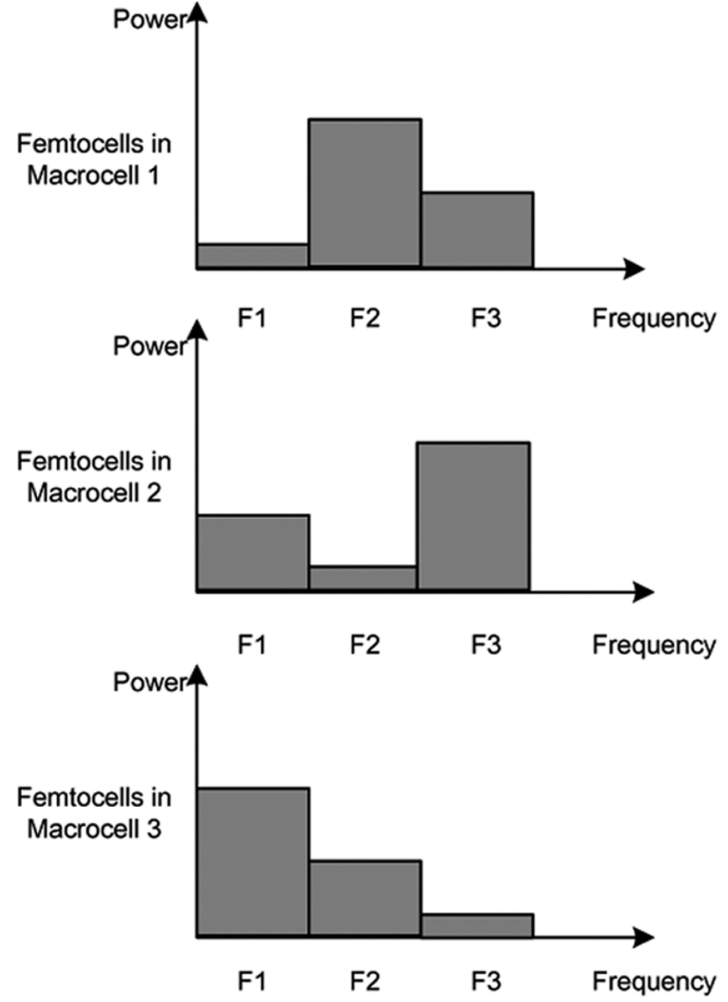

3.2) select random hopping pattern

3.3) perform frequency hopping over masked spectrum band

Additionally, to actively protect the cross-tier interference as the first priority, victim MUE detection is also performed by Femto BS in certain short duty cycle. If victim MUE in vicinity is detected ${ }^{3}$, strict Urgent Link Protection scheme will be performed as follows:

Step 4: Urgent Link Protection

4.1) reduce to low transmit power

4.2) update frequency reuse pattern

4.3) renew hopping pattern for current UEs

4.3) faster $\mathrm{FH}$ speed

However, one shall note that such victim detection is not always perfect, especially for the current state of Femtocell development. Therefore in the simulation, we assume a missdetect rate of $30 \%$ and false-alarm rate of $10 \%$.

\section{Simulations}

To validate the proposed design, we built up a simulation platform on MATLAB and conducted Monte-Carlo simulations with 1000 loops on realizations of Femtocell and user distributions ${ }^{4}$. In the simulation, we assumed a uniform distribution of Femtocells in the map and FUEs have also a uniform distribution within a $12 \times 12 \mathrm{~m}$ square of FBS's vicinity. The Macro UEs are also uniformly distributed over the whole system. All these distributions are mutually independent. The Femtocell density can be as high as 500 . Furthermore, we assume the FUE number per Femtocell is

\footnotetext{
${ }^{3}$ When FBS has the functionality of sniffing on neighboring $U L$ channel and certain detected signal strength is higher than threshold.

${ }^{4}$ Due to the computation limits, 1000 loops are basically not enough for such map size. This is the exact reason causing fluctuations on the simulation curves. Nevertheless, we can still observe the rising/descending trend of each curve.
} 


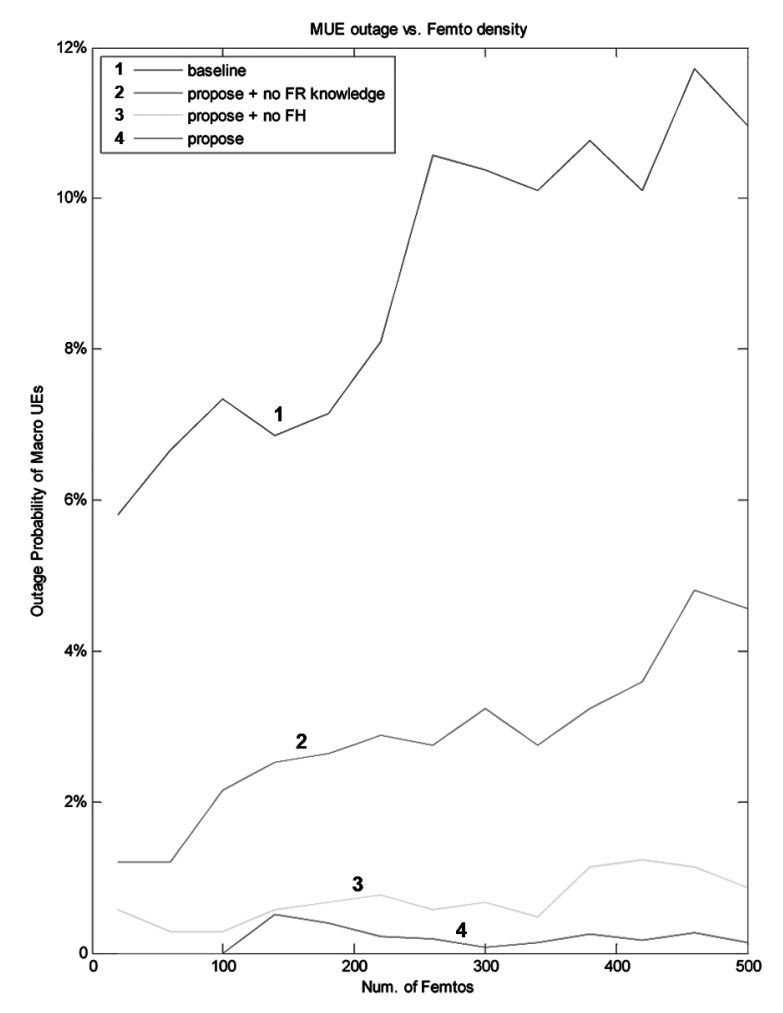

Fig.5: Simulation Results: MUE outage vs. Femto num.

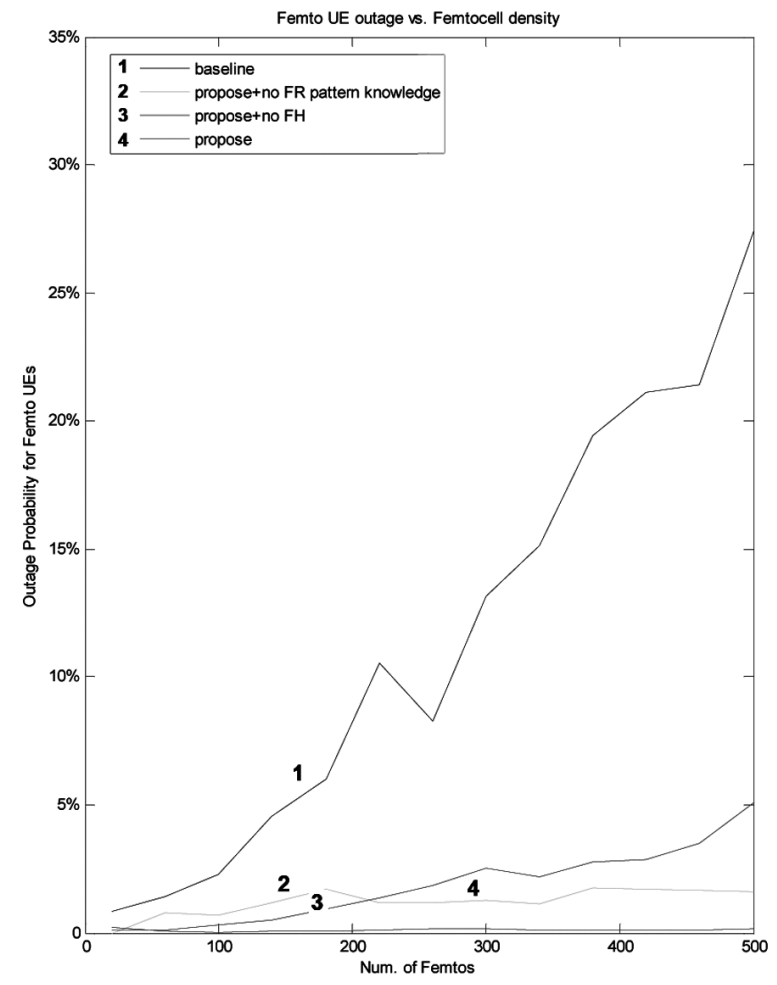

Fig.6: Simulation Results: FUE outage vs. Femto num.

only one or two and other simulation parameters can be looked up in Table 1.

To make comparisons, we assume a baseline scheme of performing no special transmission schemes for the Femto BSs. From the simulation results in Fig. 5, we can see a great improvement in Macro UEs' outage rates for our proposed scheme, meaning that the proposed scheme successfully guarantees the Macrocell links as its first priority. In Fig. 6,

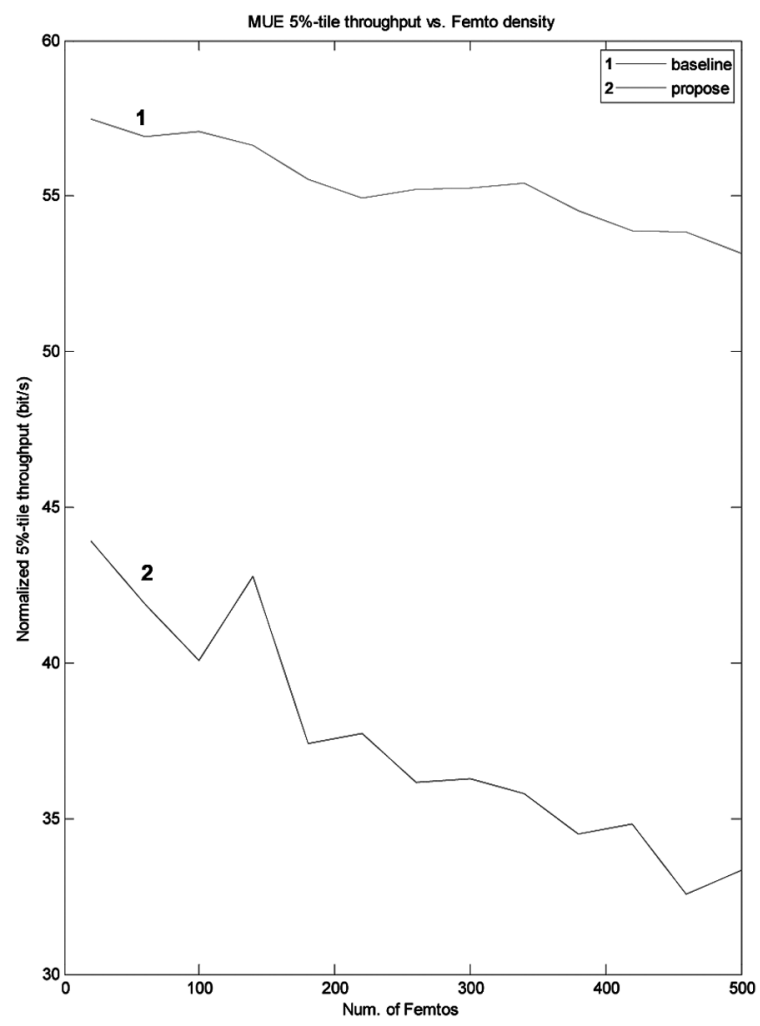

Fig.7: Simulation Results: MUE throughput vs. Femto num

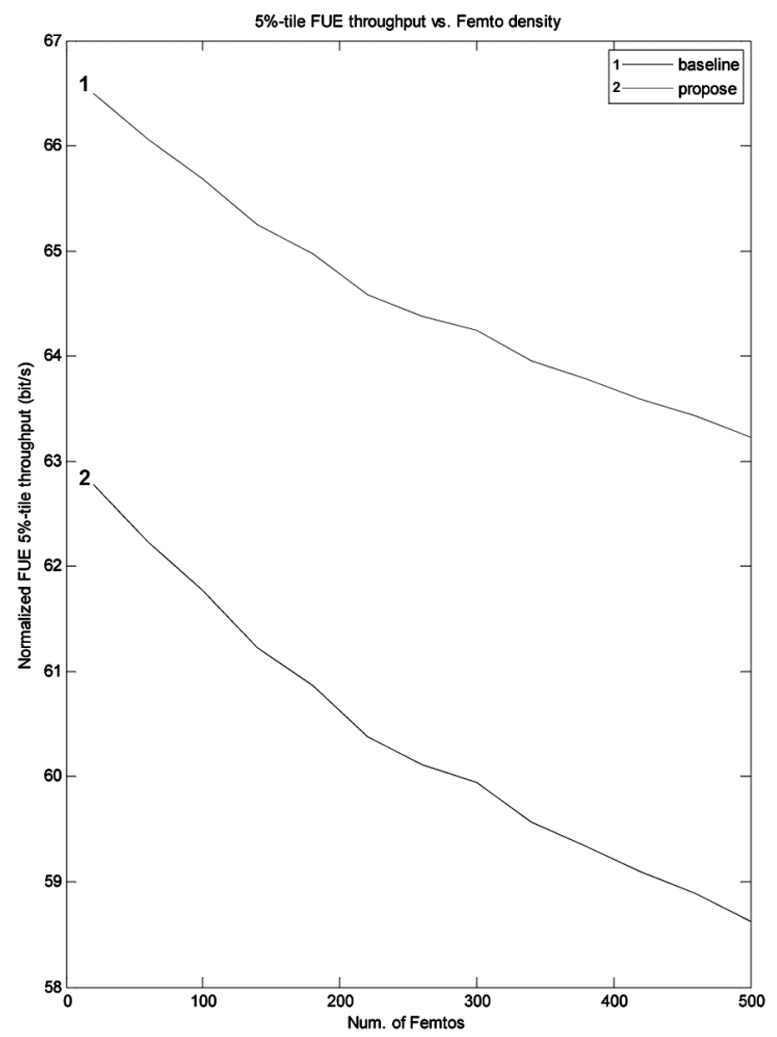

Fig.8: Simulation Results: FUE throughput vs. Femto num.

we can still observe a substantial outage improvement for Femto UEs. In Fig.7, the Macrocell's user throughput is more robust against the deployment of Femtos. And in Fig.8, due to our conservative victim MUE detection mechanics, the throughput of Femto UE declines also fast in high Femto density situation, but still better than no protection schemes. 


\begin{tabular}{|l|l|}
\hline Parameter & Used \\
\hline Cell Layout & $21 \mathrm{Hexagonal}$ sector \\
\hline Inter-Site Distance & $500 \mathrm{~m}$ \\
\hline Building Floor & 4 \\
\hline Path Loss Model 1 & $\mathrm{MBS}: P L=15.3+37.6 \log _{10} d$, din $\mathrm{m}$ \\
\hline Path Loss Model 2 & FBS: $P L=38+28 \log _{10} d$ \\
\hline Shadowing & standard deviation of $8 \mathrm{~dB}$ \\
\hline Fading & frequency selective \\
\hline Penetration Loss & $20 \mathrm{~dB}$ for out-to-indoor, $20 \mathrm{~dB}$ for floor-to-floor \\
\hline Bandwidth & $20 \mathrm{MHz}$ \\
\hline Frequency & $2 \mathrm{GHz}$ \\
\hline BS Power & $40 \mathrm{dBm}$ \\
\hline BS Antenna Gain & $15 \mathrm{dBi}$ for Macro, $0 \mathrm{dBi}$ for Femto \\
\hline UE Antenna Gain & $0 \mathrm{dBi}$ \\
\hline UE Noise Figure & $9 \mathrm{~dB}$ \\
\hline FH Pattern & Welch-Costas \\
\hline FH speed & fast: 1 symbol/hop normal: 7 symbol/hop \\
\hline link-system mapping & EESM \\
\hline Traffic Model & const-bit-rate \\
\hline
\end{tabular}

\section{Conclusions}

In this paper, while Frequency Hopping and Frequency Reuse are well-studied "classical" techniques that are commonly used in today's communication, we propose a heuristic approach of combining both to solve the interference problems in Femto-equipped DL SFR/FFR scenarios. The development of such simple but effective transmission scheme should be credited to the unique characteristics of Femtocell. Although the current solution itself is sub-optimal, by simulation it has already shown great potentials in lowering the system outage rate and improving the throughput.

Based on the observation of such potentials, it is reasonable for the further study to look for utilizing the joint Frequency Hopping and Reuse mechanics in a more optimal manner. Moreover, in the near future, we can also expect elaborate transmission designs in other scenarios of Femtocells that can also sufficiently exploit the distinctions of Femtocell. We also believe that, since the proposed transmission scheme has no specific restraints on the system degrees of freedom, it can be combined into other designs to further improve the performance of Femtocell.

\section{References}

[1] V. Chandrasekhar, et. al. "Femtocell Networks: A Survey," IEEE Communications Magazine. September 2008, vol. 46, no.9, pp. 59 67.

[2] Femto Forum, "Femtocell Business Case Whitepaper," Femto Forum White Paper, available at: www.femtoforum.org, June 2009.

[3] Femto Forum Work Group 2, "Interference Management in OFDMA Femtocells," Femto Forum White Paper, available at: www.femtoforum.org, March 2010.

[4] The 3rd Generation Partnership Project, "3G Home Node B (HNB) study item Technical Report," 3GPP R4 25.820, available at: www.3gpp.org, December 2008.

[5] V. Jungnickel, et. al. "Synchronization of Cooperative Base Stations," Proc. 2008 IEEE International Symposium on Wireless Communication Systems (ISWCS). 2008, pp. 329-334.

[6] D. Lopez-perez, et. al. "OFDMA Femtocells: A Roadmap on Interference Avoidance," IEEE Communications Magazine. September 2009, vol. 47, no. 8, pp. 41-48.

[7] V. Chandrasekhar, et. al. "Distributed Power Control in FemtocellUnderlay Cellular Networks" Proc. IEEE GLOBECOM 2009, IEEE. 2009, pp. 1-6.

[8] V. Chandrasekhar and J. Andrews "Power Control in Two-Tier Femtocell Networks" IEEE Transactions on Wireless Communications. August 2009, vol. 8, no. 8, pp. 4316-4328.

[9] V. Chandrasekhar and J. Andrews "Spectrum Allocation in Tiered Cellular Networks" IEEE Transactions on Communications. October 2009, vol. 57, no.10, pp. 3059-3068.

[10] V. Chandrasekhar and J. Andrews "Uplink Capacity and Interference Avoidance for Two-Tier Femtocell Networks" IEEE Transactions on Wireless Communications. July 2009, vol. 8, no.8, pp. 3498-3509.

[11] J. Lee, et. al. "Other-Cell Interference Reducing Resource Allocation in OFDM Based Asynchronous Cellular Systems," EURASIP Journal on Wireless Communications and Networking. January 2008, pp. $1-9$.

[12] M. Rahman, H. Yanikomeroglu, "Interference Avoidance through Dynamic Downlink OFDMA Subchannel Allocation using Intercell Coordination," Proc. IEEE Vehicular Technology VTC Spring 2008. May 2008, pp. 1630-1635.

[13] A.L. Stolyar, H. Viswanathan, "Self-organizing Dynamic Fractional Frequency Reuse in OFDMA Systems," Proc. IEEE INFOCOM 2008, IEEE, pp. 691-699.

[14] D. Gonzalez, et. al. "System Level Evaluation of LTE Networks with Semidistributed Intercell Interference Coordination," Proc. 20th IEEE International Symposium on Personal Indoor and Mobile Radio Communications, PIMRC 2009, September 2009, pp. 1497 1501.

[15] Y. Medjahdi, et. al. "Inter-cell Interference Analysis For OFDM/ FBMC Systems," IEEE 10th Workshop on Signal Processing Advances in Wireless Communications, 2009. SPAWC '09, pp. 598602.

[16] D. Tse and P. Viswanath, "Fundamentals of Wireless Communication," Cambridge University Press, May 2005.

First Author

Zhao Zhao

Institute of Communications Technology

Leibniz University of Hannover

Appelstr. 9 A, 30167, Hannover, Germany

E-mail: zhao.zhao@ikt.uni-hannover.de

Other authors

Thomas Kaiser, Andreas Wilzeck

Institute of Communications Technology

Leibniz University of Hannover

Appelstr. 9 A, 30167, Hannover, Germany 